\title{
Response of a spray of $n$-heptane or dodecane at an acoustic pressure antinode in reactive conditions
}

\author{
Clément Patat $^{* 1}$, Jean-Bernard Blaisot ${ }^{1}$, Eric Domingues ${ }^{1}$, Françoise Baillot ${ }^{1}$ \\ ${ }^{1}$ CORIA, CNRS, Normandie Université, UNIROUEN, INSA ROUEN, Saint-Étienne-du-Rouvray, \\ France \\ ${ }^{*}$ Corresponding author email: clement.patat@coria.fr
}

\begin{abstract}
Lean liquid-fueled combustion is a promising technology to reduce the environmental impact of the aeronautical industry. However, lean flames in a confined environment like an annular combustor are prone to develop thermo-acoustic instabilities, potentially leading to a decrease of combustion efficiency or even safety issues. To better understand the driving mechanisms, a collection of three swirling spray flames is aligned in a cavity, simulating an unfolded sector of an annular combustor which exhibits self-excited azimuthal instabilities of frequency $f_{r}$ about $730 \mathrm{~Hz}$. Experiments are performed with n-heptane or dodecane, and the central flame is placed at a pressure antinode of a forced transverse acoustic field at a frequency $f_{r}$. The gain of the Flame Describing Function depends strongly on the fuel, while its phase is quite unchanged. Phase-averaged spray quantities (count, velocity and size), obtained with Phase Doppler Particle Analyzer, are modulated at $f_{r}$ with an amplitude that depends on the fuel. Droplet number waves are generated alternatively in the inner region and the outer region of the spray. This pattern induces equivalence ratio fluctuations, the amplitude of which depends on the fuel. The increased presence of small droplets with $\mathrm{n}$-heptane compared to dodecane suggests that they dominate the flame response.
\end{abstract}

\section{Keywords}

Combustion instabilities, Swirling spray flames, Experiment, Transverse acoustic forcing.

\section{Introduction}

Reducing the environmental impact of energy generation is at the center of attention. This includes the development of more efficient energy systems. Disruptive technologies emerge to reach the targets in polluting emissions and fuel consumption. Among these, lean combustion is promising for aeronautical engines and gas turbines. However, lean flames are more easily subject to combustion instabilities. These phenomena result in a loss of combustion efficiency or, in extreme cases, in combustor damage. Mechanisms at the origin of combustion instabilities have been widely investigated for premixed flames subjected to a longitudinal acoustic forcing, without swirling flows [1] and with swirling flows [2, 3]. However, real engines often work with liquid fuels, and combustion instabilities are generally coupled with azimuthal modes of the combustor. The presence of a spray introduces the need for the fuel to evaporate and to mix with the air before the combustion process. In azimuthal/transverse acoustic fields, perturbations in acoustic pressure and velocity result in various flame responses, depending on the type of perturbation to which the flame is subjected. Oscillations of the heat release rate (HRR) are generally more sensitive to axial velocity fluctuations, which are induced by the pressure fluctuations at a pressure antinode of a standing transverse acoustic field [4]. Axial velocity fluctuations induce the formation of a droplet number density wave in non-reacting [5] and reacting [6] experiments, and in numerical studies [7]. This may lead to the formation of local clusters of droplets, potentially reducing the local evaporation rate [8]. The drop size distribution also fluctuates during the acoustic cycle [9], in particular through the high sensitivity of small droplets to axial velocity fluctuations [10]. These features result in the formation of an equivalence ratio wave, which leads to an oscillation of the HRR. Moreover, the quality of the atomization was found to be an important parameter in self-excited combustion instabilities [11]. A numerical work has shown that combustion instabilities can be driven by the unsteady evaporation rate of fuel droplets [12]. The 
coupling between acoustics and combustion is often quantified via the Flame Describing Function (FDF) framework. As stated by the Rayleigh criterion, in-phase pressure perturbations and HRR oscillations feed an acoustic mode of the cavity. However, unstable combustor behaviour depends on the efficiency of this coupling, which needs to be high enough to surpass damping effects. This efficiency is quantified by the gain of the FDF. A small decrease of the gain can avoid the system to become unstable [13]. A study with n-heptane and dodecane shows the fuel-dependence of the gain and phase of the FDF, which reflects a less efficient coupling and a slightly longer time delay between acoustics and combustion with dodecane [14]. This study aims to highlight the impact of the liquid fuel nature on the thermoacoustic response of swirling spray flames. N-heptane and dodecane are chosen due to unlike characteristic evaporation times. First, the experimental setup and diagnostics are presented. Secondly, the spray and its capability to follow the air flow are characterized without acoustics. Then, the flame response to an acoustic pressure perturbation is examined, and finally the spray response to the acoustic perturbation is evaluated.

\section{Material and methods}

The experimental setup is made of an acoustic cavity composed of a fixed base, two movable side walls and a set of front walls of various lengths, allowing to change the length of the cavity, $L_{c}$. Here, $L_{c}=880 \mathrm{~mm}$ is fixed, as well as the width $e=55 \mathrm{~mm}$ and height $h_{c}=200 \mathrm{~mm}$ of the cavity. A convergent piece of inox steel of height $60 \mathrm{~mm}$ and exit width $10 \mathrm{~mm}$ is placed on top of the cavity. A linear array of five injectors is placed inside the cavity. Each injector is constituted of a plenum, a co-rotating radial swirler, a distributor and a convergent nozzle of exit diameter $D_{\text {exit }}=8$ $\mathrm{mm}$, through which the air flows. The three central injectors feature a simplex atomizer of liquid fuel located at a distance $0.67 D_{\text {exit }}$ upstream the nozzle exit. The two side injectors are only supplied in air (see Fig. 1). They stabilize the lateral flames, in order to put the central flame in an environment similar to that encountered in an annular combustor. This setup simulates an unfolded sector of the annular combustor MICCA-Spray, which presents self-excited azimuthal instabilities [15]. The acoustic standing transverse 2T1L mode of the cavity of resonance frequency $f_{r}$ is forced by a couple of compression drivers Beyma CP850ND fixed on the side walls. Here, attention is put on the behaviour of a flame located at a pressure antinode (PAN). Analysis of the flame response to acoustics is performed with a photomultiplier (PM), equipped with a filter centered on $\lambda=305 \mathrm{~nm}$ with a FWHM of $24 \mathrm{~nm}$ to observe emission of the $\mathrm{OH}^{*}$ radical, which is linked to the HRR. Spray analysis is performed in reactive conditions with a two-component Phase Doppler Particle Analyzer (PDPA) system, which allows to obtain the fuel velocity components $U_{r}^{f}, U_{\theta}^{f}, U_{z}^{f}$ and the diameter of the fuel droplets. The two couples of laser beams emitted at $488 \mathrm{~nm}$ and $532 \mathrm{~nm}$ are focused with an achromatic lens of focal length $250 \mathrm{~mm}$. The reception probe, equipped with a lens of focal length $500 \mathrm{~mm}$, is placed at $30^{\circ}$ with respect to the axis of the beams. Measurements are performed during 4 seconds for around 40 measurement locations distributed along a line cutting the axis of the injector. They are carried out at a height above the injector $\hat{z}=z / D_{\text {exit }}=0.26$. Fuel is $\mathrm{n}$-heptane or dodecane. The operating points for both fuels share the same air and fuel mass flow rates, and $f_{r}$ has similar values (see Table 1). Therefore, the air flow features are not modified. Superscripts ' $a$ ' and ' $f$ ' denote air and fuel respectively. The flame power of $5.9 \mathrm{~kW} /$ flame was chosen from a previous study and shows a strong flame response with n-heptane, together with the possibility to reach high levels of acoustic forcing [6]. Raw measurements of the droplet properties (count, velocity and size) are phase-averaged in order to determine their evolution during a reconstructed acoustic cycle. The cycle is divided in 20 bins of equal duration, $1 /\left(20 f_{r}\right)$. In the following, phase-averaged quantities are enclosed by $\langle\cdot\rangle$.

\begin{tabular}{c|cccccc}
\hline Fuel & $\begin{array}{c}\text { flame power } \\
(\mathrm{kW})\end{array}$ & $\begin{array}{c}\text { equivalence } \\
\text { ratio }\end{array}$ & $\begin{array}{c}\dot{m}^{f} \\
(\mathrm{~g} / \mathrm{s})\end{array}$ & $\begin{array}{c}\dot{m}^{a} \\
(\mathrm{~g} / \mathrm{s})\end{array}$ & $\begin{array}{c}U_{\text {bulk }} \\
(\mathrm{m} / \mathrm{s})\end{array}$ & $\begin{array}{c}f_{r} \\
(\mathrm{~Hz})\end{array}$ \\
\hline $\begin{array}{c}\text { n-heptane } \\
\text { dodecane }\end{array}$ & 5.9 & 0.85 & 0.13 & 2.35 & 38.9 & $719-732$
\end{tabular}

Table 1. Operating points and experimental characteristics. 

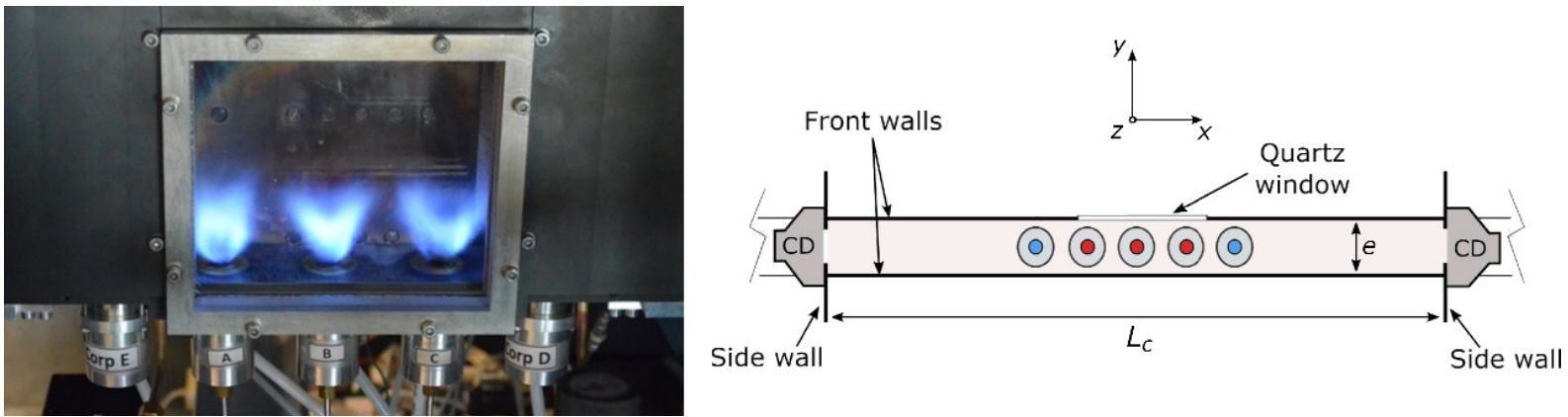

Figure 1. Left: photography of the three flames for $n$-heptane. The plenums of the five injectors are visible under the cavity base. Right: top view of the acoustic cavity. Here, $L_{c}=880 \mathrm{~mm}$ and $e=55 \mathrm{~mm}$. Red circles: injectors delivering liquid fuel and air; blue circles: injectors delivering air only. CD: compression driver.

\section{Results and discussion}

\section{Characterization of the sprays without acoustics}

Radial profiles of droplet diameter $\left(D_{10}, D^{r m s}\right)$ and vertical velocity $\left(\overline{U_{z}^{f}}, U_{z}^{f, r m s}\right)$ and of local Stokes number are presented in Fig. 2 for $n$-heptane and dodecane without acoustics at $\hat{z}=z / D_{\text {exit }}=0.26$. The profiles of $D_{10}$ and $D^{r m s}$ calculated on all the droplets present an asymmetry for both fuels inherent to this injector. The spray of $n$-heptane presents smaller droplets than that of dodecane at every radial location $\hat{r}=r /\left(D_{\text {exit }} / 2\right)$. Near the injector axis, the difference in $D_{10}$ and $D^{r m s}$ is particularly noticeable: values with dodecane are almost twice as high as those with n-heptane. The drop size distributions of the whole spray measured by PDPA are presented in Fig. 2 (f) for $n$ heptane and dodecane without acoustics at $\hat{z}=0.26$. The main mode of both normalized drop size distributions is around $d=10 \mu \mathrm{m}$. The maximum droplet diameter is around $30 \mu \mathrm{m}$ for $\mathrm{n}$-heptane and $50 \mu \mathrm{m}$ for dodecane. Higher values of $D_{10}$ for dodecane, coupled with a higher density and boiling point than $\mathrm{n}$-heptane, suggest a longer droplet evaporation time. These characteristics may influence the overall delay between fuel injection and combustion. However, for both fuels, the spray mainly consists in small droplets.

$\overline{U_{z}^{f}}$ and $U_{z}^{f, r m s}$ are calculated for the complete droplet population and for diameter classes $C_{n}$ defined by diameters $d$ satisfying $5(n-1)<d(\mu \mathrm{m}) \leq 5 n$ with $1 \leq n \leq 4$, except for $C_{5}$ constituted of droplets of diameter $d>20 \mu m$. Data are reported in Fig. 2 only for class $C_{1}$ and for n-heptane for clarity reason, but profiles for a given class $C_{n}$ are similar for the two fuels. The velocity profiles calculated on all the droplets present an asymmetry not observed when diameter classes are considered, indicating that the asymmetry observed for all droplets result from the asymmetry in the drop size distributions observed in $D_{10}$ profile. $C_{1}$ profile shows a good agreement with those calculated on all the droplets. Low values of velocity, even negative sometimes, are found for $C_{1}$ droplets as well as for all droplets in the region near the injector axis, indicating the rise of the central recirculation zone, which helps stabilize the flame via the recirculation of hot gases close to the injector. Thus, measuring fuel droplet velocity gives a reasonable description of the air flow velocity. This is quantified by the

Stokes number $S t$, which evaluates their ability to follow the air flow: $S t=\frac{\rho^{f} D_{10}^{2} \bar{U}_{z}^{f}}{18 \mu_{\text {air }} D_{\text {exit }}}$, with $\rho^{f}$ the fuel density and $\mu_{\text {air }}$ the air dynamic viscosity. Here, $\mu_{\text {air }}$ is calculated at $T=320 K$ for $|\hat{r}| \leq 1$ and at $T=750 \mathrm{~K}$ for $|\hat{r}|>1$. In the high velocity annular-shaped region, where $D_{10}$ values are small, $S t$ reaches its lowest values, mostly lower than 0.5 for both fuels. Near the injector axis, $S t$ varies much with the fuel due to their difference in $D_{10}$. St is also calculated for a droplet of diameter $5 \mu \mathrm{m}$, based on the vertical velocity of class $C_{1}$. The corresponding Stokes number is lower than 0.25 at all radial positions, so it can be assumed that $C_{1}$ droplets follow the air flow correctly.

\section{Flame response with $n$-heptane and dodecane}

The flame response to acoustics is quantified via the Flame Describing Function (FDF) framework:

$$
\mathbb{F}(f, \Pi)=\frac{\breve{I} / \bar{I}}{\breve{P} / \rho^{a} U_{b u l k}}=G_{\mathbb{F}}(f, \Pi) e^{i \phi_{\mathbb{F}}(f, \Pi)}
$$




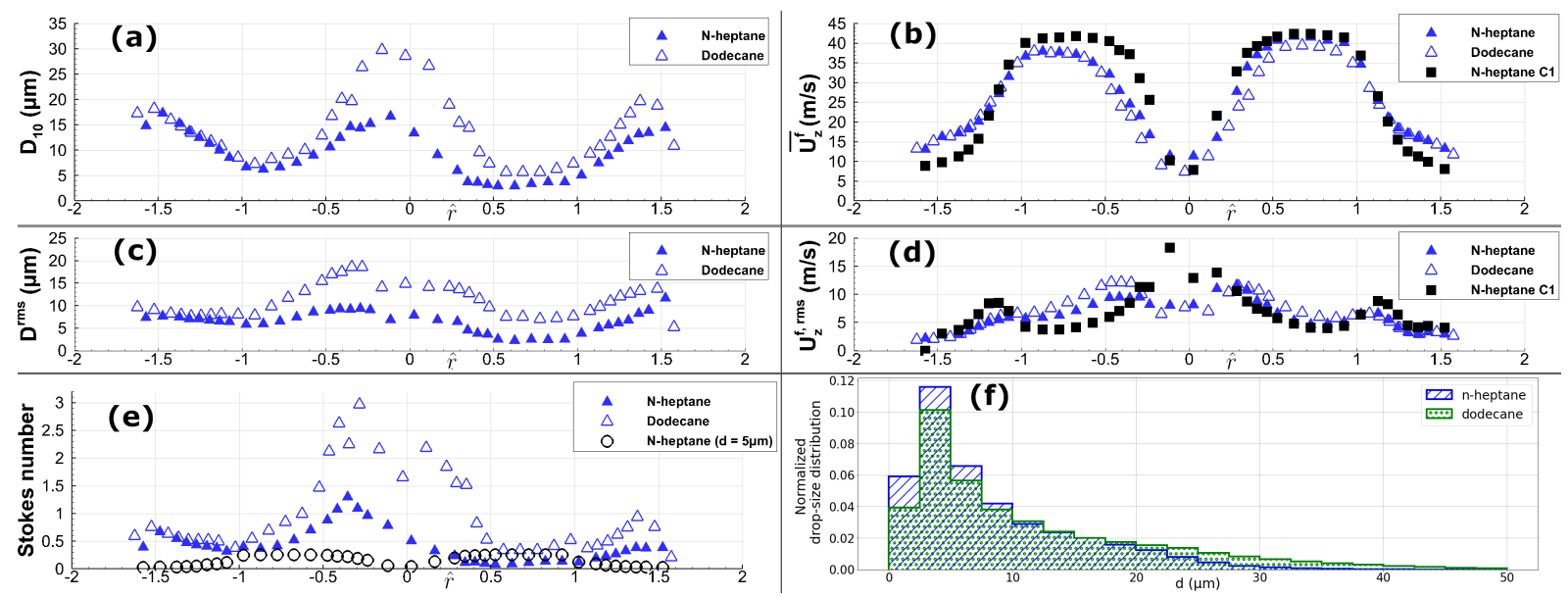

Figure 2. Radial profiles for $n$-heptane and dodecane droplets at $\hat{z}=0.26$ without acoustics: (a) mean diameter $D_{10}$; (b) mean vertical velocity $\overline{U_{z}^{f}}$; (c) rms diameter $D^{r m s}$; (d) rms vertical velocity $U_{z}^{f, r m s}$; (e) Stokes number. Normalized fuel drop size distribution: (f). Filled and empty triangles: all droplets; square: class $C_{1}$, circle: $d=5 \mu \mathrm{m}$.

where $\Pi=P_{r m s} / \rho^{a} U_{b u l k}^{2}$ is a non-dimensional parameter, $P_{r m s}$ is the rms acoustic pressure amplitude, $\rho^{a}$ the air density, $U_{\text {bulk }}$ the bulk velocity calculated at the nozzle exit and $I$ the $\mathrm{OH}^{*}$ intensity of the flame. $\breve{P}$ and $\breve{I}$ are the Fourier transforms of the $\mathrm{OH}^{*}$ intensity oscillation $I^{\prime}$ and the pressure perturbation $P^{\prime}$ respectively. The gain $G_{\mathbb{F}}$ quantifies the efficiency of the coupling between combustion and acoustics and the phase $\phi_{\mathbb{F}}$ expresses the phase shift between $P^{\prime}$ and $I^{\prime}$. Therefore, $\phi_{\mathbb{F}}$ includes the time delays introduced by all the processes between $P^{\prime}$ and $I^{\prime}$, namely response of the injector to the acoustic perturbation, convection of the perturbation to the flame region, evaporation of the liquid fuel and mixing with the air, and finally combustion. However, it was noted from a Large Eddy Simulation performed with n-heptane that evaporation occurs simultaneously with convection [16], thus $\phi_{\mathbb{F}}$ cannot be expressed just by the addition of the delays induced by all the processes. FDFs are presented for both fuels in Fig. 3. $G_{\mathbb{F}}$ is around twice higher for n-heptane than for dodecane, indicating a more efficient coupling between acoustics and combustion for $n$-heptane. Nevertheless, as $\Pi$ increases, it follows the same evolution for both fuels: a plateau up to a transition zone starting at $\Pi_{\text {trans }}=0.3$ followed by a linear decrease up to $\Pi \simeq 0.6$. Therefore, increasing the amplitude of the acoustic perturbation above $\Pi_{\text {trans }}$ results in a loss of the efficiency of the combustion-acoustics coupling. $\phi_{\mathbb{F}}$ is quite unchanged for the two fuels, indicating that it may be dominated by convective effects that are similar for both fuels. To understand the variation of the gain with the fuel, the sprays are firstly characterized without acoustics, then their response to the acoustic perturbation is examined.
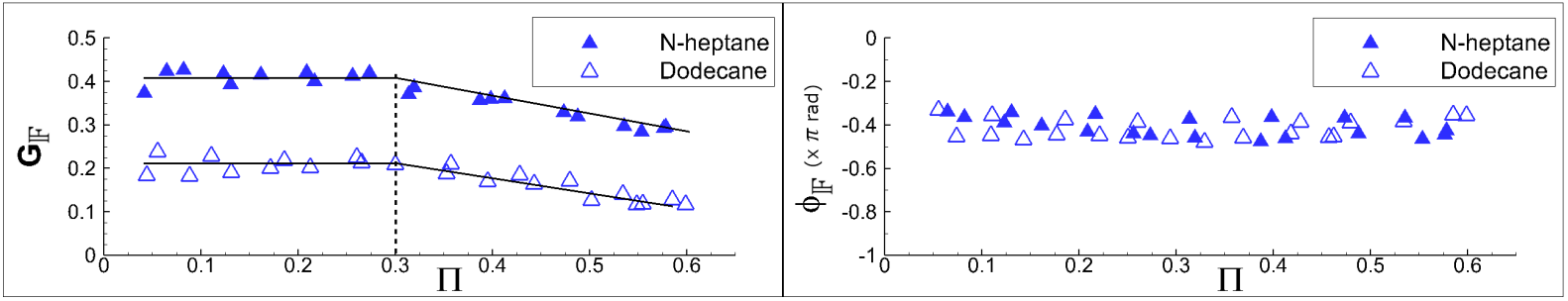

Figure 3. Flame Describing Function for n-heptane and dodecane. Left: gain $G_{\mathbb{F}}$; right: phase $\phi_{\mathbb{F}}$. Dash line: $\Pi_{\text {trans }}=0.3$.

\section{Characterization of the sprays with acoustics Generation of a droplet number wave with acoustics}

The evolution of the phase-averaged droplet count $\langle N\rangle(\hat{r}, \alpha)$, where $\alpha$ is the cycle phase angle, is illustrated for both fuels at $\hat{z}=0.26$ and for $\Pi=0.22$ in Fig. 4, with the mean droplet count without acoustics $\overline{N_{0}}(\hat{r})$. To compare the measurements, $\overline{N_{0}}(\hat{r})$ is averaged during the same duration $1 /\left(20 f_{r}\right)$ as $\langle N\rangle(\hat{r}, \alpha)$. Grey color indicate zones with a population lower than 0.04 droplet during $1 /\left(20 f_{r}\right)(e . g$. 
110 droplets per measurement without acoustics), which is too low to validate the corresponding measurements. Without acoustics, the sprays of $n$-heptane and dodecane present a core empty of droplets, which extends from the injector axis to $\hat{r} \simeq 0.4$. Then, $\bar{N}_{0}(\hat{r})$ increases sharply to reach a plateau that ranges from $\hat{r}=0.6$ to $\hat{r}=1.2$, which corresponds to a region where most of the droplets are found. Finally, $\bar{N}_{0}(\hat{r})$ decreases sharply between $\hat{r}=1.2$ and $\hat{r}=1.35$, above which an external region empty of droplets is observed.

Acoustics induces a radial oscillation of the envelop of the sprays, which is similar in phase for both fuels. The boundary of the core oscillates between $\hat{r}=0.3$ at the pressure minimum, and $\hat{r}=0.6$ at the pressure maximum, but the empty core becomes populated during $50 \%$ of an acoustic cycle for dodecane and only $30 \%$ for $n$-heptane. The population of the external region is more uniform and its boundary oscillates much less. Moreover, an oscillation of $\langle N\rangle$ is induced, giving rise to an inhomogeneous liquid-fuel distribution over space and time, which in turn generates space-time fluctuations of equivalence ratio. The oscillation amplitude of $\langle N\rangle$, which is higher with $\mathrm{n}$-heptane than with dodecane, depends much on $\hat{r}$. Consequently, the liquid-fuel distribution evolves with time such that the density of droplets oscillate with a greater amplitude in the inner region of the spray, $\hat{r}_{-}(\hat{r} \leq 1)$ than in its outer region, $\hat{r}_{+}(\hat{r}>1)$. This suggests that effects of the spray dynamics on the flame response are mainly induced by the inner region of the spray.

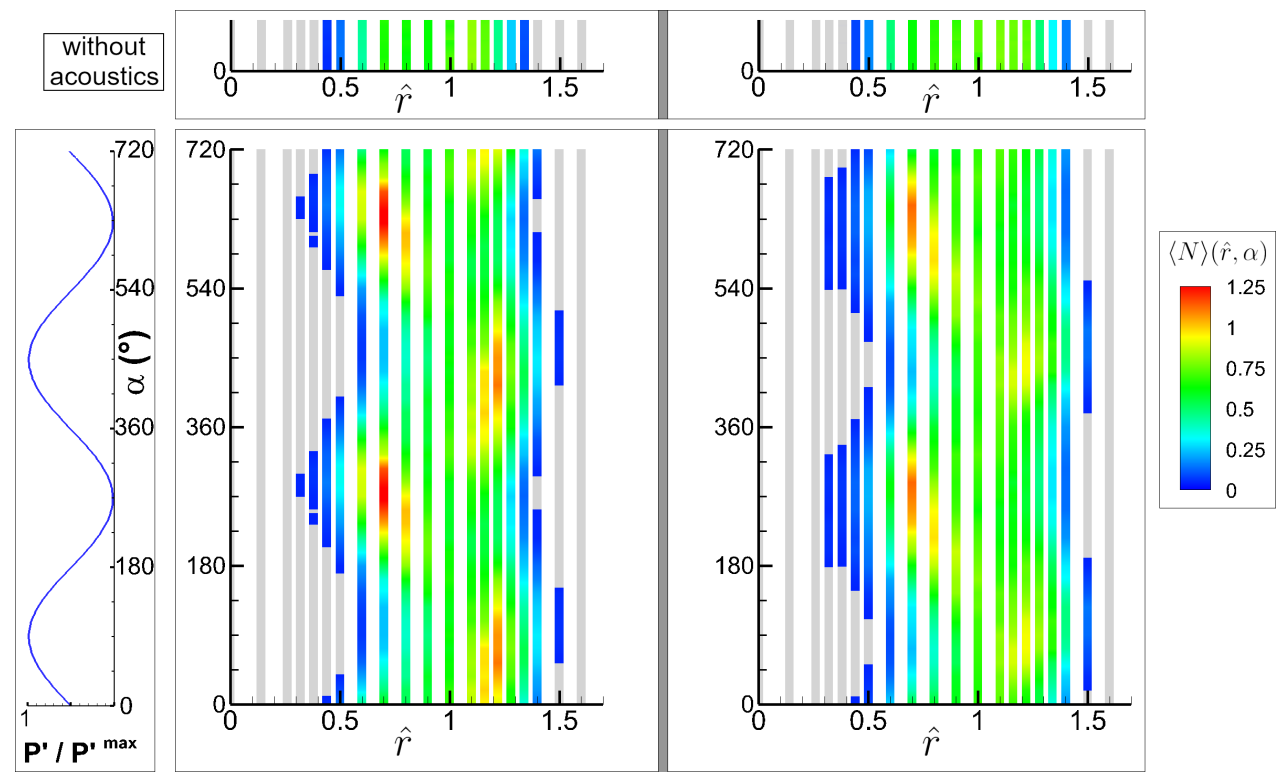

Figure 4. Phase-averaged droplet count $\langle N\rangle(\hat{r}, \alpha)$ over two reconstructed pressure cycles along a radius at $\hat{z}=0.26$ for $\Pi=0.22$. Left: $\mathrm{n}$-heptane; right: dodecane. Grey: $\langle N\rangle \leq 0.04$ droplet/cycle.

To create an oscillation of the HRR, the equivalence ratio fluctuations need to be transported to the flame zone. Fig. 5 shows the phase-averaged droplet vertical velocity, $\left\langle U_{z}^{f}\right\rangle(\hat{r}, \alpha)$, at $\hat{z}=0.26$, for $\mathrm{n}$-heptane and dodecane. At any phase angle, $\left\langle U_{z}^{f}\right\rangle$ is always higher in $\hat{r}_{-}$than in $\hat{r}_{+}$. Moreover, it oscillates distinctly and in phase with $\langle N\rangle$ in $\hat{r}_{-}$, while it oscillates very weakly in $\hat{r}_{+}$. Therefore, $\hat{r}_{-}$is characterized by the injection of many rapid droplets at the pressure minimum, followed by a few droplets that are less rapid at the pressure maximum. Segregation effects (i.e. faster droplets reaching the slower ones), could occur and enhance the equivalence ratio fluctuations in the flame zone. Thus, the oscillation amplitude of $\left\langle U_{z}^{f}\right\rangle$ is of interest to estimate a potential effect of droplet segregation on the flame response. With $\mathrm{n}$-heptane, the oscillation amplitude of $\left\langle U_{z}^{f}\right\rangle$ can increase by $40 \%$ compared to dodecane: for example, at $\hat{r}=0.6,\left\langle U_{z}^{f}\right\rangle$ oscillates between $35.7 \mathrm{~m} / \mathrm{s}$ and $48.4 \mathrm{~m} / \mathrm{s}$ for $\mathrm{n}$-heptane and between $33 \mathrm{~m} / \mathrm{s}$ and $42.1 \mathrm{~m} / \mathrm{s}$ for dodecane. This feature is observed in the entire $\hat{r}_{-}$region. Therefore, inhomogeneity in spatial drop distribution is reinforced with $\mathrm{n}$ heptane and may participate in the stronger flame response compared to that with dodecane. In $\hat{r}_{+}$region, the weak fluctuation of $\left\langle U_{z}^{f}\right\rangle$ for both fuels indicates that inhomogeneity is not significant in this region. However, $\left\langle U_{z}^{f}\right\rangle$ is generally weaker than in $\hat{r}_{-}$. As a consequence, the dense cloud 


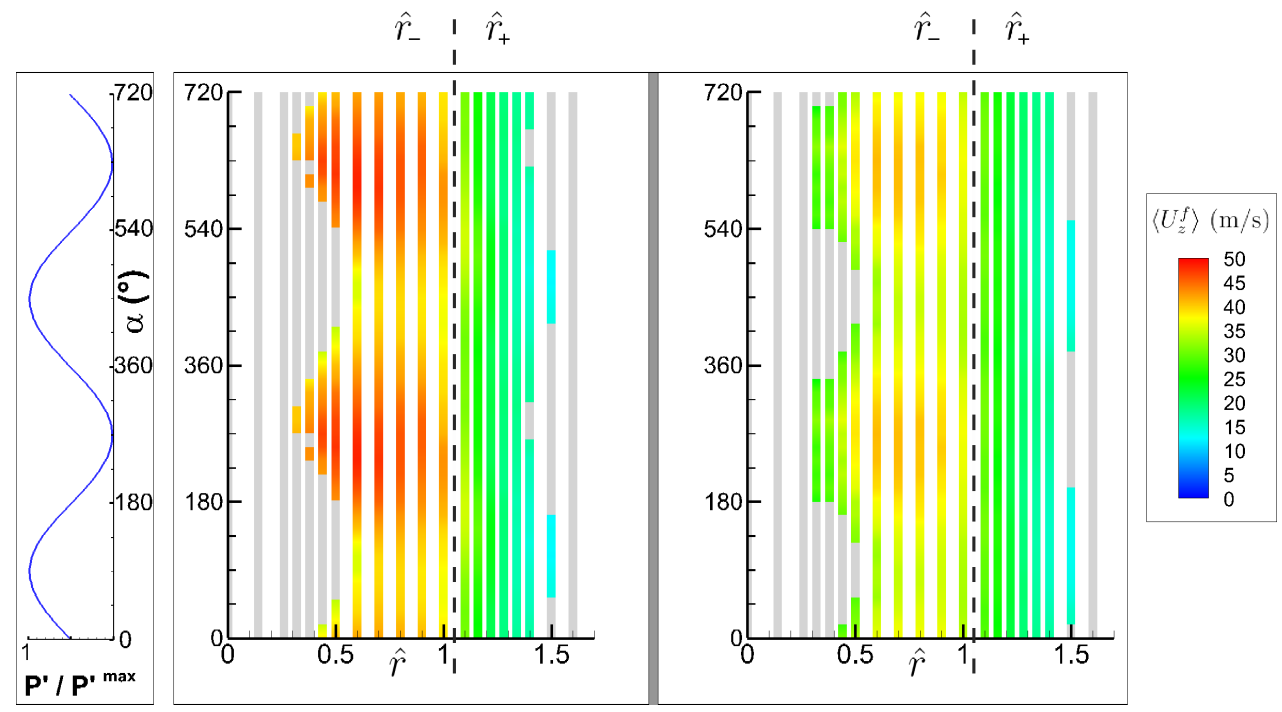

Figure 5. Phase-averaged fuel droplet axial velocity $\left\langle U_{z}^{f}\right\rangle$ over two reconstructed pressure cycles along a radius at $\hat{z}=0.26$ for $\Pi=0.22$. Left: $n$-heptane; right: dodecane. Grey: $\langle N\rangle \leq 0.04$ droplet/cycle.

of droplets, injected in $\hat{r}_{+}$near the pressure maximum, needs more time to reach the flame zone than the dense cloud of droplets injected in $\hat{r}_{-}$near the pressure minimum. Therefore, oscillations of $\langle N\rangle$ that were in phase opposition at $\hat{z}=0.26$ may interfere constructively for $\hat{z}>0.26$ due to their different propagation velocity, creating stronger equivalence ratio fluctuations in the flame zone. As the oscillation of $\langle N\rangle$ is weaker with dodecane than with $\mathrm{n}$-heptane in $\hat{r}_{-}$and $\hat{r}_{+}$, this effect could participate in the lower gain of the FDF for dodecane.

\section{Size-dependent behaviour of the droplet wave}

The phase shift $\phi_{\mathbb{E}}$ is quite similar for $n$-heptane and dodecane. As shown above, this means that the time delay between the generation of the droplet number wave at the injector exit and its combustion is close for both fuels. The relevance of $\left\langle U_{z}^{f}\right\rangle$ to estimate the propagation velocity of the droplet number wave is now discussed. Fig. 6 shows size-velocity correlations integrated throughout the measurement line. Both sprays are polydisperse but the drop size distribution for dodecane is wider since there is a population of diameters $d \geq 30 \mu \mathrm{m}$, whereas it is quasi-inexistent for n-heptane. The range of vertical velocity values covered by droplets of diameter $d$ decreases when $d$ increases, showing the greater capability of the smallest droplets to follow the velocity fluctuations of the air flow, contrary to the biggest droplets that have a quasi-ballistic behaviour. It is interesting to note that droplets of the same diameter have similar values of $U_{z}^{f}$ for both fuels, suggesting that the difference of $\left\langle U_{z}^{f}\right\rangle$ in $\hat{r}_{-}$(see Fig. 5) between $\mathrm{n}$-heptane and dodecane is mainly due to a difference in drop size distribution, instead of a reduction in vertical velocity of all droplets. This means that several droplet number waves are actually generated with various propagation velocities which decrease when $d$ increases. However, the closeness of $\phi_{\mathbb{F}}$ for the two fuels suggests that the flame response is mainly dominated by droplets of the same size, which are transported to the flame zone in an identical time. In addition, the amount of droplets with a diameter lying in the range 0-5 $\mu \mathrm{m}$ depends considerably on the fuel as it is reduced by more than $30 \%$ from n-heptane to dodecane. Consequently, $G_{\mathbb{F}}$ may be linked to the number of small droplets: since they are less numerous with dodecane, the equivalence ratio fluctuations they generate are weaker than with $n$-heptane.

The phase-averaged drop size distributions can bring information to evaluate the amplitude of the droplet number waves for different values of $d$. They are presented in Fig. 7 for both fuels, in $\hat{r}_{-}$ and in $\hat{r}_{+}$. The oscillation amplitude of $\langle N\rangle(\hat{r}, \alpha, d \leq 5 \mu \mathrm{m})$ is more important with $\mathrm{n}$-heptane than with dodecane, both in $\hat{r}_{-}$and in $\hat{r}_{+}$. However, these small droplets are much more numerous in $\hat{r}_{-}$than in $\hat{r}_{+}$. Indeed, for both fuels, they practically disappear in $\hat{r}_{+}$when $\alpha$ is in the range $220-320^{\circ}$, which corresponds to the phase angle at which they are massively injected in $\hat{r}_{-}$. Consequently, the flame response seems to be dominated by small droplets injected in $\hat{r}_{-}$. Thus, the atomization quality is an 
important parameter in the thermoacoustic coupling. The greater reactivity of small droplets to flow fluctuations make them susceptible to transform acoustic perturbations in HRR oscillations easily. However, the spray quality has a little influence on the phase of FDF.
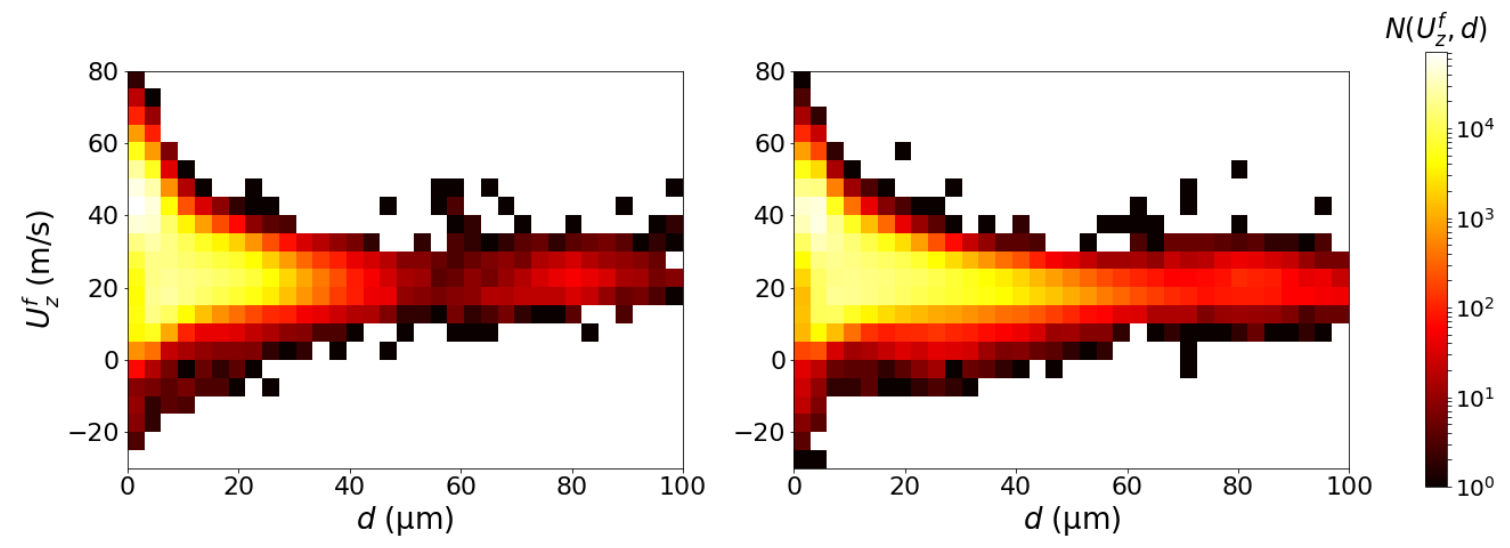

Figure 6. Fuel drop size-velocity correlations at $\hat{z}=0.26, \Pi=0.22 . N\left(U_{z}^{f}, d\right)$ indicates the number of droplets in each class of width $3 \mu \mathrm{m}$ and height $5 \mathrm{~m} / \mathrm{s}$. Left: $\mathrm{n}$-heptane; right: dodecane.
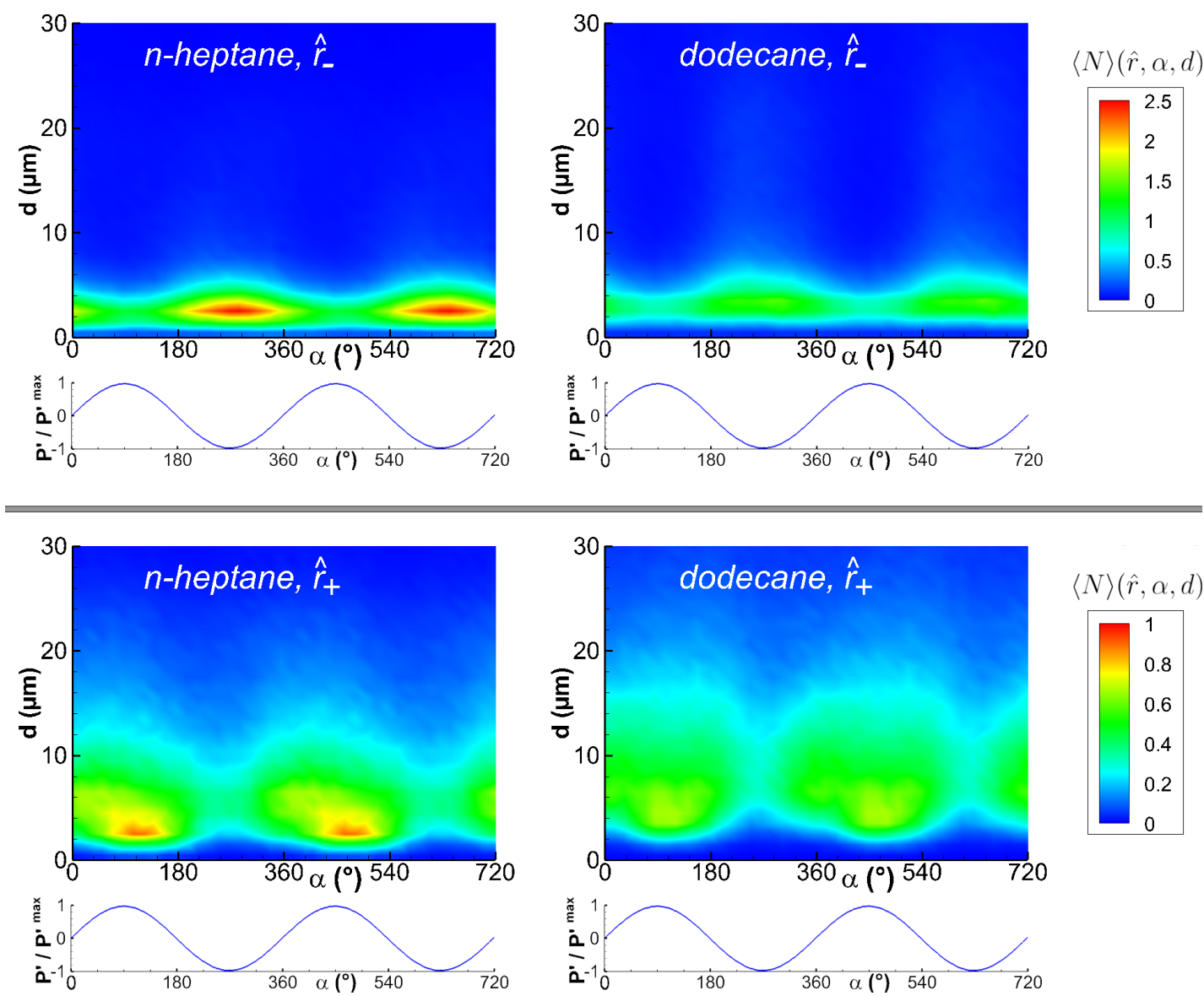

Figure 7. Phase-averaged drop size distribution $\langle N\rangle(\hat{r}, \alpha, d)$ at $\hat{z}=0.26$ for $\Pi=0.22$. Left: $\mathrm{n}$-heptane fuel; right: dodecane fuel; top: inner region of the spray, $\hat{r}_{-}$; middle: outer region of the spray, $\hat{r}_{+}$; bottom: two reconstructed acoustic cycles.

\section{Conclusion}

Acoustic forcing and spray analysis were performed on a linear array of swirling spray flames for $\mathrm{n}$-heptane and dodecane. The flame response of the central flame, which was placed at a pressure antinode of the transverse acoustic field, was studied in the Flame Describing Function (FDF) framework. The gain of the FDF is greater with n-heptane, while its phase is quite insensitive to the fuel 
used. For both fuels, an injection pattern is induced by acoustics at the injector exit, which consists in the alternative injection of many rapid and small droplets in the inner region of the spray, $\hat{r}_{-}$, and slower and bigger droplets in the outer region of the spray, $\hat{r}_{+}$. This pattern generates equivalence ratio fluctuations which are more intense in $\hat{r}_{-}$, and in phase opposition between $\hat{r}_{-}$and $\hat{r}_{+}$. Segregation effects can transform this destructive interference in a constructive one in the flame zone. The characteristics of the droplet number waves generated in $\hat{r}_{-}$and $\hat{r}_{+}$also depend on the fuel: in particular, their amplitude is more intense with $n$-heptane. The same phase shift of the FDF for $\mathrm{n}$-heptane and dodecane suggests that the flame response is induced by droplets of a similar size for both fuels, which are transported from the injector exit to the flame zone in an identical time. The higher count of small droplets and its stronger modulation with $n$-heptane than with dodecane indicates that the flame response may be dominated by small droplets.

\section{Acknowledgements}

This work was supported by the French National Research Agency (ANR) through the project FASMIC ANR-16-CE22-0013, Région Normandie and French Ministry of Higher Education and Research. The authors wish to thank RENADIAG for the technical support provided to this research.

\section{References}

[1] Balachandran, R., Ayoola, B.O., Kaminski, C.F., Dowling, A.P., Mastorakos, E., 2005, Combustion and Flame, 143, pp. 37-55.

[2] Bellows, B.D., Bobba, M.K., Forte, A., Seitzman, J.M., Lieuwen, T., 2007, Proceedings of the Combustion Institute, 31, pp. 3181-3188.

[3] Palies, P., Durox, D., Schuller, T., Candel, S. 2010, Combustion and Flame, 157, pp. 1698-1717.

[4] O'Connor, J., Acharya, V., Lieuwen, T., 2015, Progress in Energy and Combustion Science, 49, pp. 1-35.

[5] Gajan, P., Strzelecki, A., Platet, B., Lecourt, R., Giuliani, F., 2007, Journal of Propulsion and Power, 23 (2), pp. 390-397.

[6] Baillot, F., Patat, C., Caceres, M., Blaisot, J.-B., Domingues, E., 2021, Proceedings of the Combustion Institute 38(4), pp. 5987-5995.

[7] Achury, J. and Polifke, W., 2017, The Journal of Computational Multiphase Flows, 9(1), pp. 32-46.

[8] Réveillon, J., Demoulin, F.X., 2007, Proceedings of the Combustion Institute, 31, pp. 2319-2326.

[9] Kumara Gurubaran, R. and Sujith, R.I., 2011 International journal of spray and combustion dynamics, 3 (1), pp. 1-22.

[10] Giuliani, F., Gajan, P., Diers, O., Ledoux, M., 2002, Proceedings of the Combustion Institute, 29, pp. 91-98.

[11] de la Cruz Garcia, M., Mastorakos, E., Dowling, A.P., 2009, Combustion and Flame, 156, pp. 374-384.

[12] Duvvur, A., Chiang, C.H., Sirignano, W.A., 1996, Journal of Propulsion and Power, 12 (2), pp. 358-365.

[13] Mejia, D., Selle, L., Bazile, R., Poinsot, T., 2015, Proceedings of the Combustion Institute, 35, pp. 3201-3208.

[14] Rajendram Soudararajan, P., Vignat, G., Durox, D., Renaud, A., Candel, S., June 22.-26. 2020, Proceedings of the ASME Turbo Expo, GT2020-15123.

[15] Vignat, G., Durox, D., Renaud, A., Candel, S., 2020, Journal of Engineering for Gas Turbines and Power, 142(1), pp. 011016.

[16] Vignat, G., Lo Schiavo, E., Laera, D., Renaud, A., Gicquel, L., Durox, D., Candel, S., 2021, Proceedings of the Combustion Institute, 38(4), pp. 6015-6024. 